Basrah Journal

Of Surgery

\title{
EVALUATION OF SEPTAL EXTENSION GRAFT IN RHINOPLASTY
}

\section{HUSAM M ALAAMER}

MB,ChB, FICMS, Specialist Plastic Surgeon, Al-Sadr Teaching Hospital, Basrah, IRAQ.

\begin{abstract}
Septal extension graft is a unique technique for controlling nasal lengthening and tip projection, rotation, and shape by which a graft is fixed to the septum, leading to a strong and stable supporting structure. Nasal tip three-dimensional positioning is a vital and difficult component in the overall appearance of the tip.

A total of 12 Middle Eastern patients with thick nasal envelope skin were subjected to rhinoplasty using the septal extension graft (SEG) method.

Eleven out of the 12 patients, had pleasing results after primary rhinoplasty with SEG, while only one patient had SEG as a secondary procedure.

In conclusion, septal extension graft in rhinoplasty is an effective technique for achieving a strong supporting force of the nasal tip for adequate projection.

Keywords: Nasal tip, surgery, septal extension, graft, rhinoplasty.
\end{abstract}

\section{Introduction}

One of the greatest challenges in aesthetic rhinoplasty is the overly thick nasal skin envelope as thick nasal skin is a major impediment to cosmetic refinement of the nose. For Middle East individuals with thick nasal skin envelope, it is important to achieve longterm stable rhinoplasty results with less complication rates.

Although nasal tip correction procedures comprise an important part of rhinoplasty, they can be problematic because the support structures of the nasal tip may be weak and complex, and disruption of accompanying nasal attachments may cause changes to the shape of the tip making it difficult to predict the final postoperative shape $e^{1,2}$. This is especially true for Asian patients, who tend to have thick skin and weak underdeveloped cartilages, therefore techniques for nasal tip correction is limited in this patient population $^{3}$. The problem is in the determination of the lateral view intraoperatively which is important to avoid excessive extension and can cause caudal displacement of the columella, leading to hanging columella. In addition, positioning the graft under the dome and tapering it by a tip-defining suture would prevent the caudal end of the graft from extruding into the interdomal space which will be broaden eventually ${ }^{4}$. because of this problem, septal extension graft method is performed for this type of rhinoplasty.

\section{Patients and methods}

From January 2015 through October 2018, a total of 12 Middle Eastern patients with thick nasal skin envelope were subjected to rhinoplasty using the SEG method. Male to female ratio was $1: 2$ and age ranged from 22 to 54 years (mean age 38 years).

Anthropometric Measurements: to evaluate the outcomes, measurement was done for nasal tip projection (defined as the straight line from the soft tissue at the base of the columella to the greatest projection point of the tip-dorsal 
intersection at a standard lateral view of the nose) and nasolabial angle (formed by the labial surface of the upper lip at the midline and the inferior border of the nose) preoperatively and postoperatively at 3 months and 1 year intervals.

Surgical Technique: Surgery was performed under general anesthesia for all the patients. Local infiltration of anesthetic solution consisted of lidocaine $1 \%$ with 1:200000 epinephrine was injected into mucoperichondrium of the septum and the nose. In total, 5 to $10 \mathrm{mls}$ of local anesthetic solution was used for infiltration of the nose, including the dorsal skin, nasal floor, and the septum. Open rhinoplasty was performed in all the cases. The skin flap was elevated by linking a columellar incision and an infracartilaginous incision. When the incision was performed along the caudal margin of the lower lateral cartilage, it was applied up to the most lateral part of the lateral crus. The elevation of the skin flap was conducted widely to make redraping of the stretched cartilage structures possible without tension. The nasal septal mucoperichondrium was elevated bilaterally to make mucoperichondrial pockets for insertion of the SEG. The septal cartilage was harvested by using a caudal septal approach, leaving a 8-12 $\mathrm{mm}$ L-shaped strut, depending on the thickness of the septum. When septal cartilage was lacking or inadequate especially in those with cleft lip nasal deformity, autologous rib cartilage was harvested. Rib cartilage was designed as $2 \mathrm{~cm}$ long, $2 \mathrm{~cm}$ wide, and 1.5 to $2 \mathrm{~mm}$ thick on a grid plate. The prepared cartilage graft was inserted into the mucoperichondrial pocket of the septum and fixed to the septum or to the spreader cartilage graft with 5-0 PDS sutures at 3 or 4 points. For firm fixation of the graft, the contact surface of the graft and the nasal septum was created to be around 3 to $4 \mathrm{~mm}$. The degree of septal extension depended on preoperative tip projection and rotation but generally consisted of a $10-15 \mathrm{~mm}$ intraoperative projection. After fixation of the cartilage graft, the lower lateral cartilages (LLCs) were suspended to the tip of the SEG and fixed in position with 5-0 PDS sutures. Lastly, the nasal skin was closed with 6-0 nylon sutures, Steps are shown in figures 1,2 , and 3 .

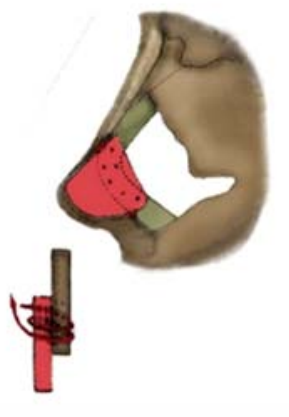

Fig.1: Fixation of SEG to septal cartilage in at least 3 to 4 points.

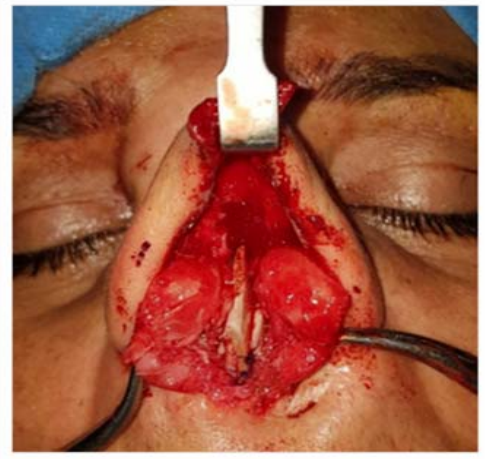

Fig.2: SEG with costal cartilage is fixed to spreader grafts and the septum

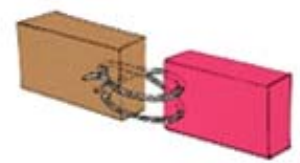

Fig.3: A vertical figure-of-eight suture yields more stability than a horizontal figure-of-eight suture. 


\section{Results}

Eleven $(92 \%)$ of the 12 patients, had satisfactory results after primary rhinoplasty with SEG, while only one case $(8 \%)$ have SEG as a secondary procedure. Two patients with cleft lip deformity were operated upon utilizing costal cartilage grafts. The costal cartilage was used in 2 males and one female in this study while the septal cartilage was utilized in 2 males and 7 females as demonstrated in table I.

Table I: The utilization of septal and costal cartilages in this study.

\begin{tabular}{|l|l|l|l|}
\hline Donor site for cartilage & Males & Females & Total \\
\hline Costal cartilage & 2 & 1 & 3 \\
\hline Septal cartilage & 2 & 7 & 9 \\
\hline Total & 4 & 8 & 12 \\
\hline
\end{tabular}

Aesthetic assessment of the thick tip skin refinement and improved projection was performed by comparing the nasolabial angle before and after surgery. An examples of cases performed in this study are shown in figures $4 \& 5$.

Case 1: A 35 years old female with incorporation of SEG in secondary rhinoplasty. Before surgery ( $\mathrm{a} \& \mathrm{c}$ ) and 17 months after surgery (b \& d). The nasolabial angle on the lateral view is increased from less than 90 degrees to about 110 degrees with adequate tip projection.
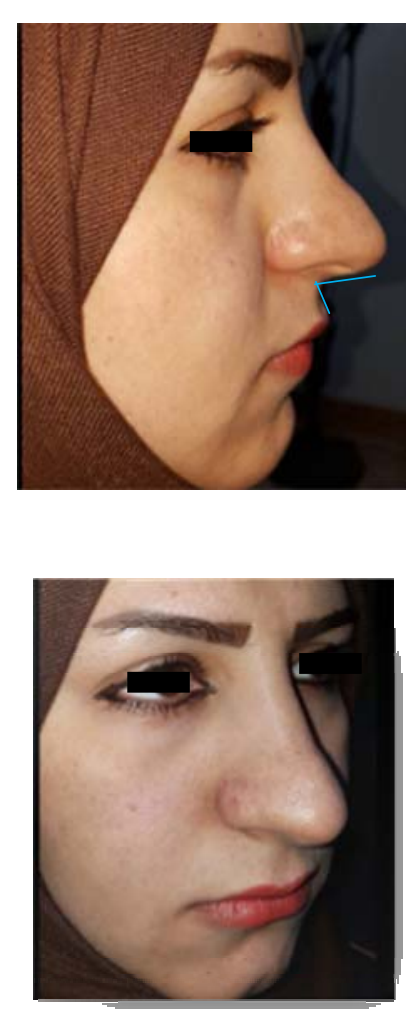
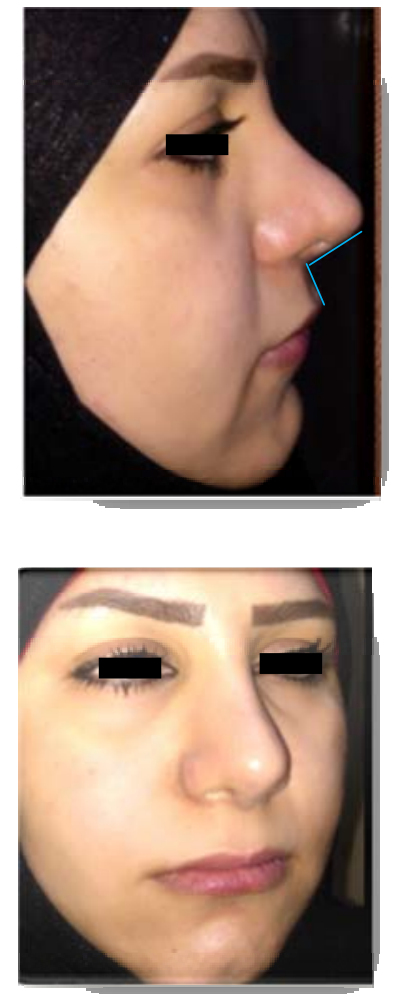

Figure 4 
Case 2: A 24 years old male with cleft lip nasal deformity requested rhinoplasty. Costal cartilage graft harvested for dorsal line alignment as spreader grafts in addition to maintaining tip projection and rotation by SEG. Before surgery (a \& c) and 14 months after surgery $(b$ \& d).
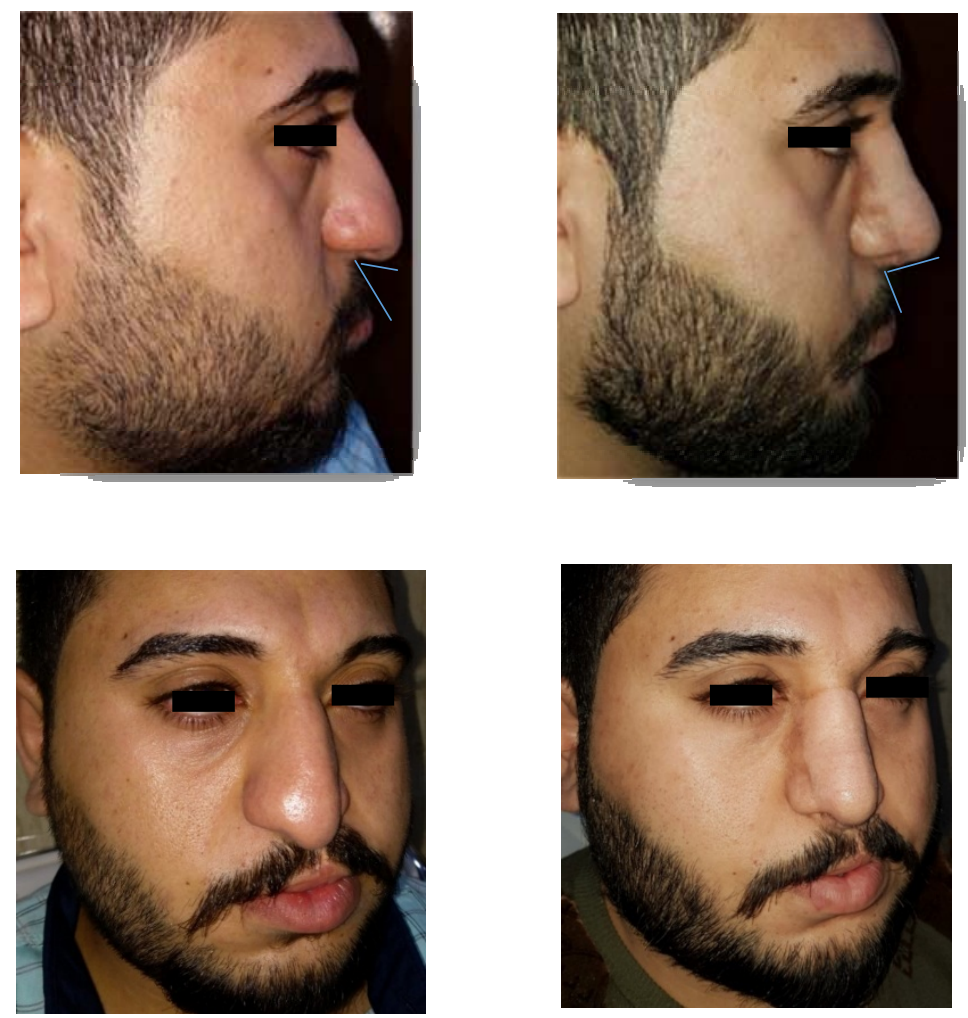

Figure 5

Complications like decrease of projection and rotation was not reported, but two cases had stiffness and one case had mild deviation of the tip as shown in table II.

Table II: Complications

\begin{tabular}{|l|l|l|}
\hline Complications & Males & Females \\
\hline Stiffness & 1 & 1 \\
\hline Deviation & - & 1 \\
\hline Decrease of projection and rotation & - & - \\
\hline Revision & - & - \\
\hline Infection & - & - \\
\hline
\end{tabular}

Patient's satisfaction was estimated by five categories after one year of the procedure confirming that most of them were satisfied as shown in table III.

\section{Table III: Patients satisfaction}

\begin{tabular}{|l|l|l|}
\hline Patients satisfaction & Males & Females \\
\hline Very satisfied & 1 & 5 \\
\hline Satisfied & 2 & 3 \\
\hline Equivocal & 1 & 0 \\
\hline Dissatisfied & 0 & 0 \\
\hline Very dissatisfied & 0 & 0 \\
\hline
\end{tabular}




\section{Discussion}

The overlapping and direct extension (butt type) grafts was investigated by Byrd et $\mathrm{al}^{5}$. The direct extension is used restrictively because of its difficulty in fixing securely, but overlapping is the most widely accepted method. The overlapping type is preferred when the graft is overlapped by more than $4 \mathrm{~mm}$, and in unilateral grafting; the direction of the graft being bent should be considered to minimize asymmetry. Han et $\mathrm{al}^{6}$ measured the shearing, buckling, and tensile force, after using a butt junction type through two simple interrupted sutures, two horizontal figure-of-eight locking sutures, and one vertical figureof-eight suture. One vertical figure-ofeight suture was the most powerful method. Compared to the butt junction type, methods contacting broadly with the cartilage such as the overlapping type may produce a more powerful fixation, but swallowing of septal mucosa and wastage of cartilage need to be taken into account. Thus, the butt junction type with the vertical figure-of-eight suture may be a good substitution in those who lack available cartilage.

Hubbard $^{7}$ described the mechanical advantage according to the direction of the septal extension graft. The spreader type has a mechanical disadvantage in tip projection because of the force vector of soft tissue resistance, but it is advantageous in nasal lengthening or derotation. In contrast, if the graft is located more perpendicularly to the nasal dorsum, it may have a mechanical advantage in tip projection. In a weak nasal supporting structure, not stable enough with only a septal extension graft, additional procedures such as a derotation graft or tip extension suture should be considered ${ }^{8}$.

Tebbetts ${ }^{9}$ described the dovetail junction in fixing the columellar strut to the septum (Fig. 6). By wedging a graft into the $3-5 \mathrm{~mm}$ gap on the caudal septum margin, strong fixation and a low possibility of asymmetry can be gained. However, it is technically difficult and only ideal when the septum is more than $1.5 \mathrm{~mm}$ thick.

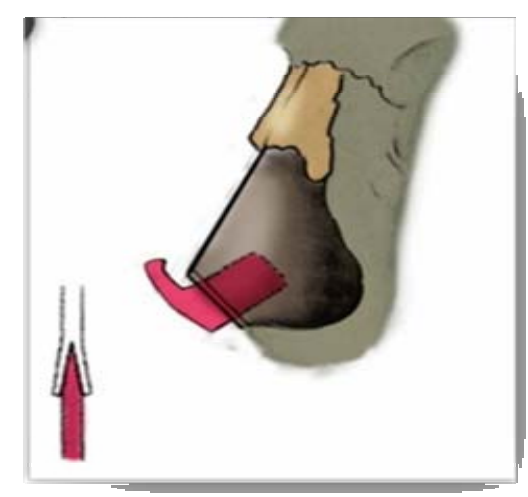

Fig. 6: Dovetail junction introduced by Tebbetts

When SEG was not sufficient for nasal tip augmentation, an additional onlay graft was performed for nasal tips that were too tense. Jin and Won $^{10}$ emphasized that an SEG and onlay graft are the main surgical methods that should be used for Asian patients. The primary source for a cartilage graft is the nasal septum; however, harvesting the septal cartilage is not always possible for a number of reasons, such as a previous septal cartilage harvest, a history of septoplasty, insufficient quantity of tissue because of anatomical characteristics, or severe ossification. In such cases, autologous rib cartilage can be considered as what is done in this study. However, it is still quite common for young female patients to refuse rib cartilage harvesting because they desire a less invasive procedure or have concerns about chest scarring. In such cases, irradiated homologous costal cartilage, ear cartilage, or Medpore grafts can be a useful alternative ${ }^{11}$.

External force can shift the cartilage grafts to either side of the caudal septum and subsequently lead to collapse and deviation of the nasal tip or failure of 
nasal elongation $^{12}$. The technique consists of a combined triangle septal extension graft and a stripped septal extension graft. The triangle septal extension graft can extend beyond the dorsal and caudal septum and the anterior septal angle. It can be applied in almost every patient who needs a septal extension graft. The placement of 2 grafts can effectively increase the stability for the nasal tip ${ }^{13}$. Conclusion; For those with a thick skin over the nasal tip like Asian and African population, utilizing septal extension graft in rhinoplasty procedure is an effective technique for achieving a strong supporting force of the nasal tip for adequate projection. There are many introduced techniques but not all problems have been fully solved, and thus they need to be assessed thoroughly.

Recommendation: The SEG is an effective way of enhancing nasal shaping for those with complex skin texture, so this study recommends utilizing it in rhinoplasty procedures.

\section{References}

1. Byrd HS, Andochick S, Copit S, et al. Septal extension grafts: a method of controlling tip projection shape. Plast Reconstr Surg. 1997;100:999-1010.

2.Tardy ME, Hendrick D, Alex J, Dayan S. Surgical anatomy of the nose. In: Calhoun KH, ed. Head \& Neck Surgery-Otolaryngology.3rd ed. Philadelphia, PA: Lippincott Williams \& Wilkins; 2001:22112227.

3.Calhoun KH. Introduction to rhinoplasty. In: Calhoun KH, ed. Head \& Neck SurgeryOtolaryngology.3rd ed. Philadelphia, PA: Lippincott Williams \& Wilkins; 2001:2229-2240.

4. Baeck SI, Joung DH, Min YG. Rhinoplasty. In: Min YG, ed. Clinical Rhinology. Seoul, Korea: Ilchokak Publishing Co; 1997:507-563.

5. Byrd HS, Ha RY. Septal extension grafts revisited: 6-year experience in controlling nasal tip projection and shape. Plast Reconstr Surg. 2003;112:1929-1935. [PubMed] [Google Scholar]

6. Han K, Jin HS, Choi TH, et al. A biomechanical comparison of vertical figure-of-eight locking suture for septal extension grafts. J Plast Reconstr Aesthet Surg. 2010;63:265-269. [PubMed] [Google Scholar]

7. Hubbard TJ. Exploiting the septum for maximal tip control. Ann Plast Surg. 2000;44:173-180. [PubMed] [Google Scholar]

8. Kim JH. Surgical guides for overcoming disadvantages of septal extension graft in Asians; 7th Asan Rhinoplasty Symposium; 2009 May 8-10; Seoul. 2009. pp. 54-57. [Google Scholar]

9. Tebbetts JB. Primary rhinoplasty: a new approach to the logic and the technicques. St. Louis: Mosby; 1998. [Google Scholar]

10.Jin HR, Won TB. Nasal tip augmentation in Asians using autogenous cartilage. Otolaryngol Head Neck Surg. 2009;140(4):526-530.PubMedGoogle ScholarCrossref

11. Suh MK, Ahn ES, Kim HR, Dhong ES. A 2-year follow-up of irradiated homologous costal cartilage used as a septal extension graft for the correction of contracted nose in Asians. Ann Plast Surg. 2013;71(1):45-49. doi:10.1097/SAP.0b013e3182468084.Google ScholarCrossref

12. Chang YL. Correction of difficult short nose by modified caudal septal advancement in Asian patients. Aesthet Surg J. 2010;30(2):166-175.PubMedGoogle ScholarCrossref

13. Jinde Lin, MD1; Xiaoping Chen, MD1; Xin Wang, MD1; et al Xia Gao, MD1; Xiangyu Zheng, MD1; Xin Chen, MD1; Yugang Yuan, MD1 JAMA Facial Plast Surg. 2013;15(5):362-368. 